The screened probe has been found to be of special value in studying the electron-energy distribution at the high-energy end. In argon the distribution has been found to fall well below the Maxwellian value at energies above 15 volts. These facts are of considerable importance in the study of the reactionrates of processes occurring in the plasma, and the study is now being extended to include electronegative gases. A fuller account of these experiments will shortly be published elsewhere.

' Langmuir and Tonks, Phys, Rev., 34, 876 (1929).

Tonks, Mott-Smith and Langmuir, Phys. Rev., 28, 104 (1926).

- Sena, L., Trans. All-Union Electrotechn. Assoc., 42 (1935).

-Sena, L., J. Phys. U.S.S.R., 10, 179 (1936).

\section{SCATTERING OF WAVES BY SPHERICAL PARTICLES}

$\mathrm{E}^{\mathrm{n}}$

VER since Tyndall's beautiful series of experiments in 1868 demonstrating the relationship between the intensity and polarization of light scattered by suspended particles and the density of the particles, and Rayleigh's subsequent deduction, on the elastic solid concept of æether vibration , that for particles of size very much smaller than the wave-length of light the intensity of scattering is proportional to the inverse fourth power of the wave-length, much interest, both theoretical and experimental, has been taken in this phenomenon of scattering. In 1908, the first rigorous treatment, according to the methods of electromagnetic theory, of the complete diffraction of a plane wave by a spherical particle of any composition was given by Gustav Mie, and this has become the classic contribution to the subject upon which subsequent discussions have been based.

Several years ago the Computation Laboratory of the United States National Bureau of Standarda applied the Mie theory to compute extensive tables of scattering functions for both real and complex values of the index of refraction of spherical particles, and these have recently been published*.

The various intensity functions, which are listed, give the angular distribution of intensity and the total light scattered as a function of $\alpha=2 \pi r / 7$, where $r$ is the radius of the small spherical particle from which the light is scattered; $r$ is approximately equal to $\lambda$, the wave-length of the incident light. From the angular distribution and the total light scattered by a suspension of particles of uniform size, the particle-size and concentration can be deduced, and the method of deduction is described. The tables are divided into four sections, the first three of which relate to real indices of refraction $m$, and the fourth to certain complex indices. In Part 1 the angular distribution functions, proportional to the intensities of the two incoherent, plane-polarized components scattered by a transparent particle illuminated with natural light, for values of $m$ between $1 \cdot 33$ and $2 \cdot 00$, and in Part 2 the total scattering coefficient, for $m=1 \cdot 50$, are tabled. From Part 3 the total scattering (and absorbing) coefficient can be obtained for absorbing materials of extinction coefficient varying between 0 and 0.1 and of $m$ between 1.44 and 1.55 .

* U.S. Department of Commerce : National Bureau of Standards. Applied Mathematical Series 4: Tables of Scattering Functions for Spherical Particles. Pp. 119. (Washington, D.C.: Government Printing Office, 1948.) 45 cents.
The sets of tables for real $m$ were computed originally on the recommendation of Prof. V. K. La Mer, professor of chemistry at Columbia University, and arose out of a series of investigations relating to chemical warfare. In Part 4 the tables have been extended to certain values of complex arguments and have application in microwave radar, where the echoes received on radar sets from suspended or falling water-droplets in the atmosphere are a particular case of the scattering problem. The range of wave-length thus considered is $10 \mathrm{~cm} .-3 \mathrm{~mm}$., and the dispersion measurements made by Dr. J. A. Saxton, of the National Physical Laboratory, are used.

An admirable foreword to the tables is contributed by Prof. J. A. Stratton, who briefly reviews the history of the problem of scattering, describes Mie's formal solution, the method by which the tables have been compiled, and their particular applications. Confidence is expressed that the tables will be of considerable benefit to physical and biological science. Mathematical definitions of the tabulated functions, as well as the physical significance of the tabulated quantities, together with a short bibliography, are given in the introduction. The tables themselves are clear and easy to read, though the size and spacing of the numbers in the tables vary almost from page to page. The paper cover is to be deplored.

\section{S. WetnTtrous}

\section{REPEATABILITY OF TEST RESULTS}

A RECENT meeting of the London Group of the A Royal Statistical Society's Industrial Applidifficulties arising from discrepancies in what are supposed to be replicate test results.

Especially in the field of chemical analysis, a great deal of empirical standardization has taken place, resulting in the publication of handbooks of standard methods of tests. These, by closely specifying the conditions of test and even in some cases the dimensions of the apparatus used, endeavour to reduce the variation experienced. It is well known, however, that in spite of these attempts at reducing variation, differences between the results of tests on the same sample made by different laboratories are experienced which are larger than those found between replicate results of the same observer or even by different observers in the same laboratory using the same apparatus.

Following the lead of the Institute of Petroleum in its Standard Methods Handbook, the names 'repeatability' and 'reproducibility' are given to measures of the two kinds of variability, between replicates of the same operator and between labor. atories. Mr. A. H. Dodd, of Newton, Chambers and Co., criticized this choice of names on the grounds of confusion, since in ordinary use the two words mean much the same. If there is any difference, it is that reproducibility implies an attempt to make two results alike, thus introducing the suggestion of faking results. He thought that the coining of new words (and he gave as an example the word 'squariate' for squared deviation) preferable, in this instance at least, to the twisting of old ones to bear new meanings. The new words would be even more acceptable if they conveyed within themselves the ideas of these two separate sources of variation. 